\title{
ERRATUM TO "HYPERFINITE AND STANDARD UNIFICATIONS FOR PHYSICAL THEORIES”
}

\author{
ROBERT A. HERRMANN
}

Received 5 January 2006; Accepted 28 March 2006

This corrects the major theorem on product consequence operators.

Copyright @ 2006 Hindawi Publishing Corporation. All rights reserved.

In [1], Definition 5.2, and Theorem 5.3 and its proof are stated incorrectly. The following is the correct definition, theorem, and proof.

Definition 5.2. Suppose one has a nonempty finite set $\mathscr{C}=\left\{C_{1}, \ldots, C_{m}\right\}$ of general consequence operators, each defined on a nonempty language $L_{i}, 1 \leq i \leq m$. Define the operator $\Pi C_{m}$ as follows: for any $X \subset L_{1} \times \cdots \times L_{m}$, using the projection $p r_{i}, 1 \leq i \leq m$, define $\Pi C_{m}(X)=C_{1}\left(p r_{1}(X)\right) \times \cdots \times C_{m}\left(p r_{m}(X)\right)$.

Theorem 5.3. The operator $\Pi C_{m}$ defined on the subsets of $L_{1} \times \cdots \times L_{m}$ is a general consequence operator and if, at least, one member of $\mathscr{C}$ is axiomless, then $\Pi C_{m}$ is axiomless. If each member of $\mathscr{C}$ is finitary and axiomless, then $\Pi C_{m}$ is finitary.

Proof. (a) Let $X \subset L_{1} \times \cdots \times L_{m}$. Then for each $i, 1 \leq i \leq m, p r_{i}(X) \subset C_{i}\left(p r_{i}(X)\right) \subset L_{i}$. But, $X \subset p r_{1}(X) \times \cdots \times p r_{m}(X) \subset C_{1}\left(p r_{1}(X)\right) \times \cdots \times C_{m}\left(p r_{m}(X)\right)=\Pi C_{m}(X) \subset L_{1} \times$ $\cdots \times L_{m}$. Suppose that $X \neq \varnothing$. Then $\varnothing \neq \Pi C_{m}(X)=C_{1}\left(p r_{1}(X)\right) \times \cdots \times C_{m}\left(p r_{m}(X)\right) \subset$ $L_{1} \times \cdots \times L_{m}$. Hence, $\varnothing \neq p r_{i}\left(\Pi C_{m}(X)\right)=C_{i}\left(p r_{i}(X)\right), 1 \leq i \leq m$, implies that $C_{i}\left(p r_{i}\right.$ $\left.\left(\Pi C_{m}(X)\right)\right)=C_{i}\left(C_{i}\left(p r_{i}(X)\right)\right)=C_{i}\left(p r_{i}(X)\right), 1 \leq i \leq m$. Hence, $\Pi C_{m}\left(\Pi C_{m}(X)\right)=\Pi C_{m}(X)$. Let $X=\varnothing$ and assume that no member of $\mathscr{C}$ is axiomless. Then each $p r_{i}(X)=\varnothing$. But, each $C_{i}\left(p r_{i}(X)\right) \neq \varnothing$ implies that $\Pi C_{m}(X) \neq \varnothing$. By the previous method, it follows, in this case, that $\Pi C_{m}\left(\Pi C_{m}(X)\right)=\Pi C_{m}(X)$. Now suppose that there is some $j$ such that $C_{j}$ is axiomless. Hence, $C_{j}\left(p r_{j}(X)\right)=\varnothing$ implies that $\Pi C_{m}(X)=C_{1}\left(p r_{1}(X)\right) \times \cdots \times$ $C_{m}\left(p r_{m}(X)\right)=\varnothing$, which implies that $C_{j}\left(p r_{j}\left(\Pi C_{m}(X)\right)\right)=\varnothing$. Consequently, $C_{1}\left(p r_{1}\right.$ $\left.\left(\Pi C_{m}(X)\right)\right) \times \cdots \times C_{m}\left(p r_{m}\left(\Pi C_{m}(X)\right)\right)=\varnothing$. Thus, $\Pi C_{m}\left(\Pi C_{m}(X)\right)=\varnothing$ and axiom (1) holds. Also in the case where at least one member of $\mathscr{C}$ is axiomless, then $\Pi C_{m}$ is axiomless.

(b) Let $X \subset Y \subset L_{1} \times \cdots \times L_{m}$. For each $i, 1 \leq 1 \leq m, p r_{i}(X) \subset p r_{i}(Y)$, whether $p r_{i}(X)$ is the empty set or not. Hence, $C_{i}\left(p r_{i}(X)\right) \subset C_{i}\left(\operatorname{pr}_{i}(Y)\right)$. Therefore, $\Pi C_{m}(X)=$ 
2 Erratum to "hyperfinite and standard unifications"

$\left.C_{1}\left(p r_{1}(X)\right) \times \cdots \times C_{m}\left(p r_{m}(X)\right) \subset C_{1}\left(p r_{1}(Y)\right) \times \cdots \times C_{m}\left(p r_{m}(Y)\right)=\Pi C_{m}(Y)\right)$ and axiom (2) holds. Thus, $\Pi C_{m}$ is, at least, a general consequence operator.

(c) Assume that each member of $\mathscr{C}$ is finitary and axiomless and let $x \in \Pi C_{m}(X)$ where, since $\Pi C_{m}$ is axiomless, $X$ is nonempty. Then for each $i, \operatorname{pr}_{i}(x) \in C_{i}\left(\operatorname{pr}_{i}(X)\right)$. Since each $C_{i}$ is finitary and axiomless, then there is some nonempty finite $F_{i} \subset p r_{i}(X)$ such that $p r_{i}(x) \in C_{i}\left(F_{i}\right) \subset C_{i}\left(p r_{i}(X)\right)$. Hence, nonempty and finite $F=F_{1} \times \cdots \times F_{m} \subset$ $\operatorname{pr}_{1}(X) \times \cdots \times p r_{m}(X)$. Then for each $i, p r_{i}(F)=F_{i}$ implies that finite $F=F_{1} \times \cdots \times$ $F_{m}=p r_{1}(F) \times \cdots \times p r_{m}(F) \subset p r_{1}(X) \times \cdots \times p r_{m}(X)$. From axiom $(2), x \in \Pi C_{m}(F)=$ $C_{1}\left(p r_{1}(F)\right) \times \cdots \times C_{m}\left(p r_{m}(F)\right) \subset \Pi C_{m}\left(p r_{1}(X) \times \cdots \times p r_{m}(X)\right)=C_{1}\left(p r_{1}(X)\right) \times \cdots \times$ $C_{m}\left(p r_{m}(X)\right)=\Pi C_{m}(X)$. This completes the proof.

\section{References}

[1] R. A. Herrmann, Hyperfinite and standard unifications for physical theories, International Journal of Mathematics and Mathematical Sciences 28 (2001), no. 2, 93-102.

Robert A. Herrmann: Institute for Mathematics and Philosophy, 44890 Rivermont Terrace 100, Ashburn VA 20147, USA

E-mail address: rah@usna.edu 


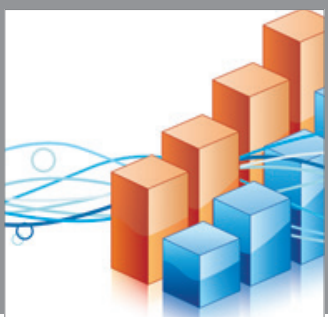

Advances in

Operations Research

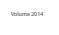

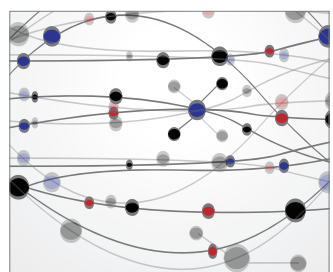

\section{The Scientific} World Journal
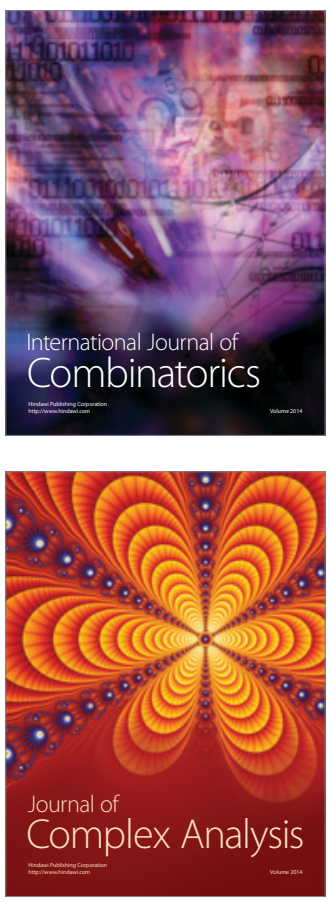

International Journal of

Mathematics and

Mathematical

Sciences
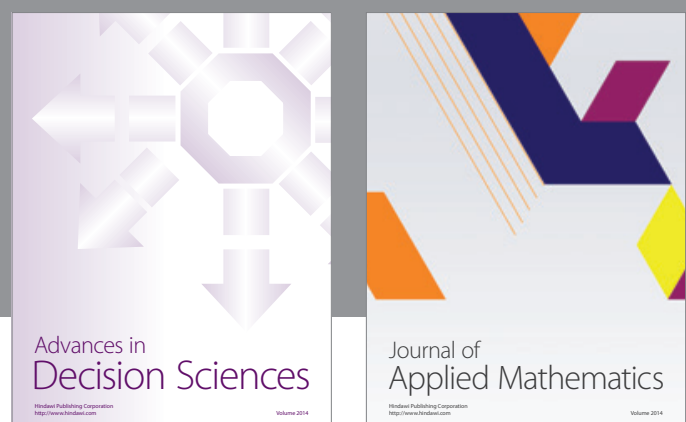

Journal of

Applied Mathematics
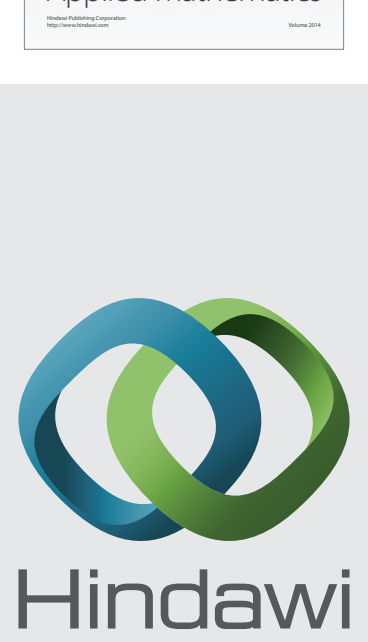

Submit your manuscripts at http://www.hindawi.com
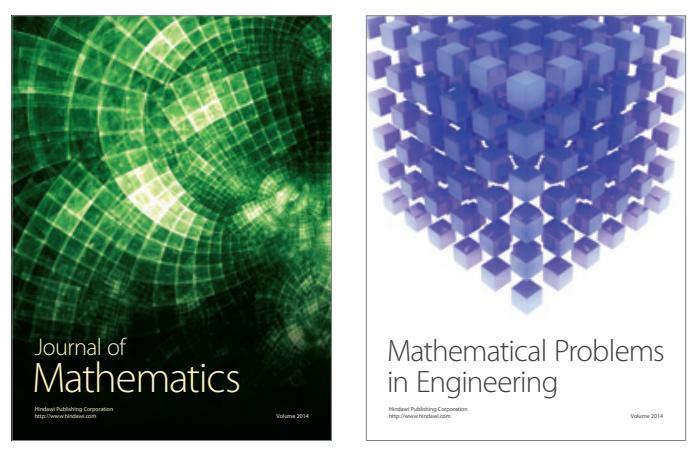

Mathematical Problems in Engineering
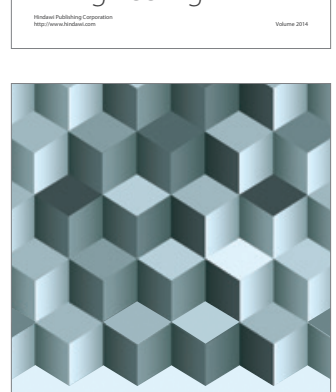

Journal of

Function Spaces
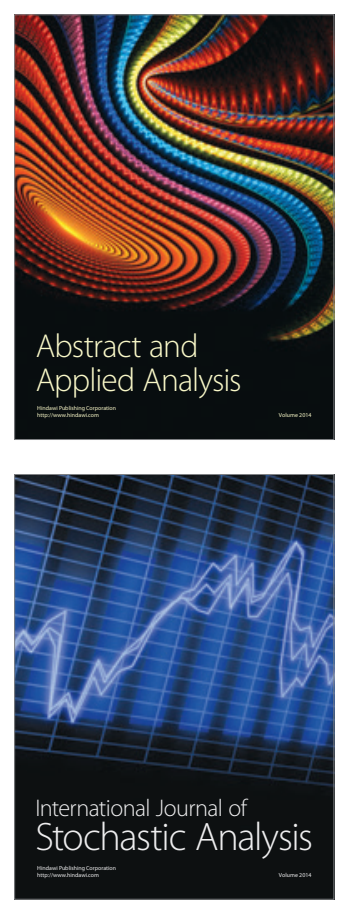

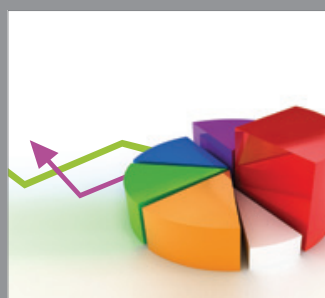

ournal of

Probability and Statistics

Promensencen
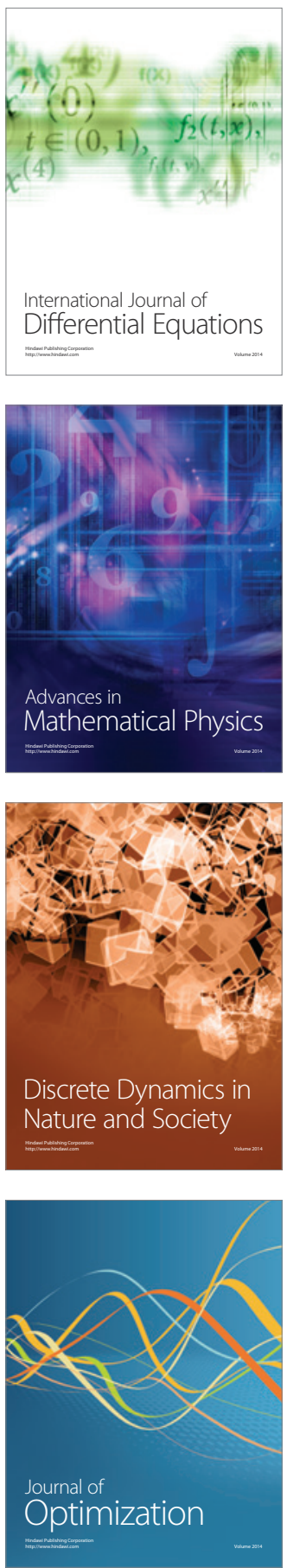